\title{
Acute Kidney Injury in a Child with Paroxysmal Cold Haemoglobinuria
}

\begin{abstract}
Paroxysmal cold haemoglobinuria $(\mathrm{PCH})$ is characterised by intravascular haemolysis mediated by the Donath-Landsteiner (D-L) antibody. It accounts for up to forty percent of all cases of autoimmune haemolytic anaemia in children. Acute kidney injury (AKI) in association with $\mathrm{PCH}$, however, is very rare with only two published case reports in the paediatric literature.

We report the case of a nine-year-old boy who developed AKI secondary to $\mathrm{PCH}$. Supportive treatment with active warming and strict fluid management was commenced. He required two sessions of haemodialysis and two warmed red cell transfusions. Recovery was complete with normal renal profile and haemoglobin at five weeks. At twenty months he remains disease-free.

This case adds to the paediatric literature describing AKI in the setting of $\mathrm{PCH}$. Although AKI is a rare complication, the importance of careful monitoring of fluid status and renal function is emphasised in the setting of acute intravascular haemolysis.
\end{abstract}

\section{Introduction}

Paroxysmal cold haemoglobinuria $(\mathrm{PCH})$, first described in 1854 , is characterized by acute intravascular haemolysis, mediated by the Donath-Landsteiner antibody. Autoantibody activation against the p-antigen on red cell membranes activates complement cascade, resulting in red cell membrane perforation and intravascular haemolysis [1]. Although rare, it accounts for upwards of $40 \%$ of all cases of autoimmune haemolytic anaemia in children [2]. Patients commonly present with haemoglobinuria, jaundice and anaemia after exposure to cold. Urticarial symptoms may occur as the p-antigen has been found on lymphocytes and skin fibroblasts [1]. Acute kidney injury (AKI) in association with $\mathrm{PCH}$, however, is very rare with only two published case reports in the paediatric literature $[3,4]$. We report the case of a 9-year-old boy who developed acute kidney injury secondary to $\mathrm{PCH}$.

\section{Case History}

A previously healthy 9 -year-old boy presented with acute onset of lip-swelling and passing dark urine, after eating an ice-lollipop. He had a preceding upper respiratory tract infection 2 weeks prior to presentation. On examination he had evidence of angioedema, was normotensive with no generalised oedema. Initial investigations revealed a normal haemoglobin level and normal renal function. Urinalysis was positive for 2+ blood. Urinary direct microscopy, however, did not reveal any red blood cells, casts or organisms.

On day 2 he deteriorated clinically with vomiting and abdominal pain. He developed jaundice, hypertension and became oliguric. Serial investigations revealed progressive acute kidney injury with intravascular haemolysis. Serum creatinine increased from a baseline

\section{Journal of}

Clinical \& Medical Case Reports

\author{
Ben McNaughten ${ }^{1 *}$, Tamara Mallett ${ }^{2}$ and Mary \\ O'Connor'
}

${ }^{\prime}$ Department of Paediatric Nephrology, Royal Belfast Hospital for Sick Children, Belfast, Northern Ireland, UK

${ }^{2}$ Department of Paediatric Nephrology, Bristol Royal Children's Hospital, Bristol, England, UK

Address for Correspondence:

Ben McNaughten, Department of Paediatric Nephrology, Royal Belfast Hospital for Sick Children, Belfast, Northern Ireland, UK, Tel: 07763607822; E-mail: bmcnaughten095@hotmail.com

\section{Submission: 11 May, 2016 \\ Accepted: 30 May, 2016 \\ Published: 04 June, 2016}

Copyright: @ 2016 McNaughten B, et al. This is an open access article distributed under the Creative Commons Attribution License, which permits unrestricted use, distribution, and reproduction in any medium, provided the original work is properly cited.

Reviewed \& Approved by: Dr. Xu Zeng, Department of Pathology and Laboratory Medicine, Temple University, USA

level of $35 \mu \mathrm{mol} / \mathrm{L}$ to a level of $233 \mu \mathrm{mol} / \mathrm{L}$ over 24 hours. Ultrasound renal tracts identified bilateral echo bright kidneys. The patient developed progressive anaemia, thrombocytopaenia, elevated LDH and bilirubin. He was managed with careful replacement of fluids, strict fluid balance and antihypertensive treatment.

Further haematological investigations confirmed a positive direct Coombs test for anti-C3d and haemoglobinuria. The diagnosis of PCH was confirmed on day 4 of admission. Initially no abnormalities were detected on blood film, but subsequent examination by the paediatric haematology laboratory revealed the unusual phenomenon of erythrophagocytosis (Figure 1).

This consumption of red cells by macrophages and other phagocytes is a rare in vivo phenomenon, pathognomonic of $\mathrm{PCH}$ [5]. It was not possible to perform the Donath-Landsteiner test in the laboratory, the gold standard diagnostic test [1], due to difficulties in transporting blood at $5{ }^{\circ} \mathrm{C}$ and $37^{\circ} \mathrm{C}$. Extensive immunology testing was otherwise normal. Creatinine kinase was normal. Rhinovirus was detected on respiratory secretions, however all other virology and

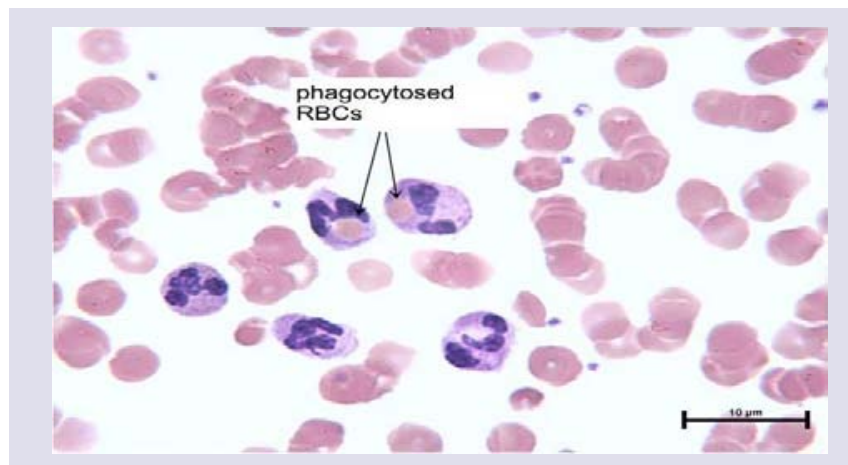

Figure 1: Blood film demonstrating erythrophagocytosis. 


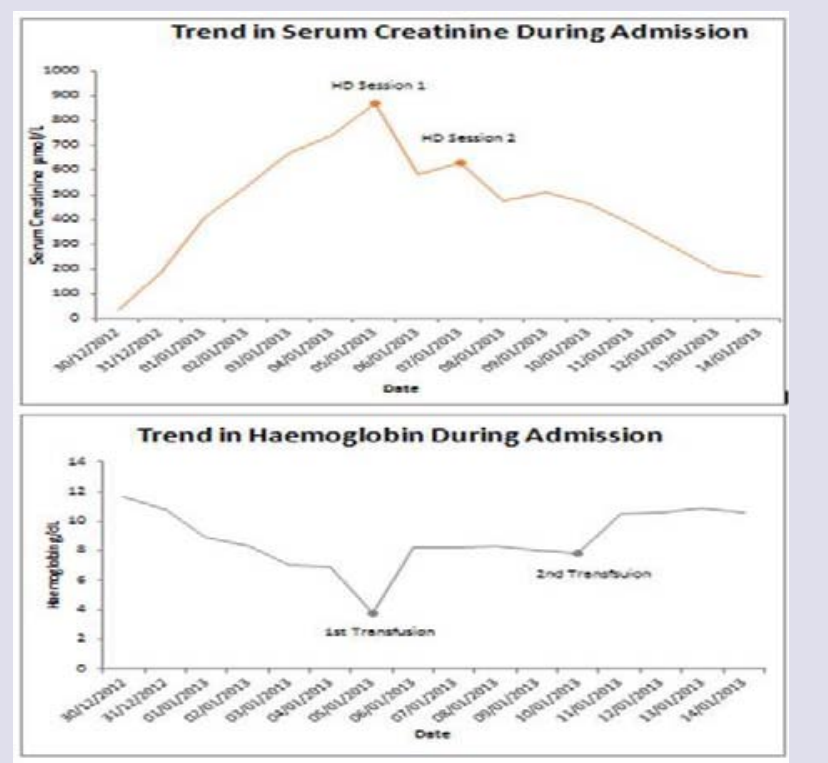

Figure 2: Graphs showing trend in serum creatinine and haemoglobin during admission.

microbiological tests were negative, including those for influenza A or B, mycoplasma, measles, EBV, parvovirus and adenovirus.

Supportive care and avoidance of cold exposure was commenced. A bear-hugger was applied and IV fluids were warmed. Nutrition was supported with feeding and a low potassium diet. He required two warmed packed red cell transfusions for treatment of anaemia on days 7 and 12 (Figure 2). In view of progressive AKI he had a temporary internal jugular venous catheter inserted and received two sessions of haemodialysis on day 7 and 9 (Figure 2).

With supportive management the patient recovered well, with resolution of haemolyisis and improvement in renal function. He was discharged on day 16 with normal haematinics and moderately impaired renal function. Avoidance of cold exposure was advised for 3 months. Renal function normalized after 5 weeks and at 20 months he remains disease-free. Long-term prognosis is felt to be excellent.

\section{Discussion}

This case report highlights an interesting case of acute kidney injury in the setting of $\mathrm{PCH}$.

$\mathrm{PCH}$ is an autoimmune haemolytic anaemia mediated by the Donath-Landsteiner antibody. The exact aetiology of the DonathLandsteiner antibody is unknown however there is a close relationship in observed cases between exposure to viral agents and the development of PCH within 2-3 weeks. Aetiological theories include the role of viral stimulation of abnormal clones of immunologically competent cells; structural alteration of the RBC membrane by a virus; and alteration of $\mathrm{T}$ suppressor function that results in autoantibody production [1]. PCH is caused by autoantibody activation after cold exposure and subsequent warming. Autoantibodies act against the p-antigen on red cell membranes causing activation of complement and resulting in red cell membrane perforation and intravascular haemolysis on warming [1]. It therefore follows that patients present like our case with haemoglobinuria, jaundice and anaemia after exposure to cold, in addition to urticarial symptoms related to $\mathrm{p}$-antigen presence on skin fibroblasts $[1,2]$. The preceding viral illness in this case may have been rhinovirus which was detected in respiratory secretions. In particular tests for Influenza $\mathrm{A}+\mathrm{B}$, Mycoplasma, Measles, EBV, Parvovirus, Adenovirus and Coronavirus were all negative. Cold exposure from ingestion of the ice-lollipop has triggered the antibody activation leading to local angioedema and haemoglobinuria. Importantly, the latter was misdiagnosed as haematuria until urine microscopy was negative for red blood cells and casts on repeat testing.

When PCH is clinically suspected, a diagnosis is made by performing the Donath-Landsteiner antibody test, an In vitro assay for biphasic intravascular haemolysis [1]. Unfortunately our laboratory did not have the appropriate facilities to perform this investigation. It was considered unnecessary, however, as the erythrophagocytosis in association with the haemoglobinuria and positive anti-C3d on the direct Coombs test was considered diagnostic of $\mathrm{PCH}[2,5]$. We emphasise the role of the paediatric haematology scientific officers in recognizing abnormalities of blood film which was misreported as normal on repeat tests in the haematology laboratory.

Treatment of $\mathrm{PCH}$ revolves around supportive care, avoidance of cold exposure and early recognition and treatment of complications. Warmed packed red cell transfusions should be administered for life threatening haemolysis and symptomatic anaemia [3], as was necessary for our patient. AKI is a very rare complication, however our patient required two sessions of haemodialysis in the management of severe uraemia, with subsequent gradual improvement in renal function. There is some evidence to support the use of plasma exchange therapy with 5\% albumin in $\mathrm{PCH}$ whilst steroids, although frequently used, have not been shown to reduce the clinical course.

Complications associated with $\mathrm{PCH}$ include urticarial eruptions from skin fibroblast activation, severe anaemia following acute intravascular haemolysis, AKI and multiorgan failure [1]. Mortality is rare and is often secondary to mulitorgan failure from severe anaemia [1]. Acute kidney injury in the setting of $\mathrm{PCH}$ is very rare with only 2 previous case reports in the paediatric literature $[3,4]$. The pathological processes by which acute kidney injury occurs in $\mathrm{PCH}$ and other forms of autoimmune haemolysis are poorly understood. Acute kidney injury, however, must be recognized as an important complication of $\mathrm{PCH}$ and other causes of acute intravascular haemolysis. It therefore follows that these patients should have strict fluid balance and renal function monitoring throughout the period of their illness.

The long term prognosis following $\mathrm{PCH}$ is excellent. It is thought that antibodies may persist in this condition for up to three months and hence cold avoidance is important during this period. The patient described in this case recovered well and remains disease free at 20 months since diagnosis.

This case adds to the paediatric literature describing the rare occurrence of AKI secondary to intravascular haemolysis caused by $\mathrm{PCH}$. Timely recognition of $\mathrm{PCH}$ allows prompt, supportive therapy. We encourage the support of paediatric haematology scientific officers in diagnostic difficulties. The importance of strict monitoring of fluid balance \& renal function in acute intravascular haemolysisis 
Citation: McNaughten B, Mallett T, O'Connor M. Acute Kidney Injury in a Child with Paroxysmal Cold Haemoglobinuria. J Clin Med Case Reports. 2016;3(1): 3 .

ISSN: $2332-4120$

is emphasised.

\section{References}

1. Slemp SN, Davisson SM, Slayten J, Cipkala DA, Waxman DA (2014) Two case studies and a review of paroxysmal cold hemoglobinuria. Lab Med 45: 253-258

2. Sokol RJ, Hewitt S, Stamps BK (1982) Autoimmune haemolysis associated with Donath-Landsteiner antibodies. Acta Haematol 68: 268-277.
3. Hothi DK, Bass P, Morgan M, Acharya J, Humphries SE, et al. (2007) Acute renal failure in a patient with paroxysmal cold haemoglobinuria. Pediatr Nephrol 22: 593-596.

4. Vergara LH, Mota MC, Sarmento Ada G, Duarte CA, Barbot JM (2006) Acute renal failure secondary to paroxysmal cold haemoglobinuria. An Pediatr (Barc) 64: 267-269.

5. Mukhopadhyay S, Keating L, Souid AK (2003) Erythrophagocytosis in paroxysmal cold haemoglobinuria. Am J Hematol 74: 196-197. 